\title{
Low cloud properties influenced by cosmic rays
}

\author{
Marsh, Nigel; Svensmark, Henrik
}

Published in:

Physical Review Letters

Link to article, DOI:

10.1103/PhysRevLett.85.5004

Publication date:

2000

Document Version

Publisher's PDF, also known as Version of record

Link back to DTU Orbit

Citation (APA):

Marsh, N., \& Svensmark, H. (2000). Low cloud properties influenced by cosmic rays. Physical Review Letters, 85(23), 5004-5007. https://doi.org/10.1103/PhysRevLett.85.5004

\section{General rights}

Copyright and moral rights for the publications made accessible in the public portal are retained by the authors and/or other copyright owners and it is a condition of accessing publications that users recognise and abide by the legal requirements associated with these rights.

- Users may download and print one copy of any publication from the public portal for the purpose of private study or research.

- You may not further distribute the material or use it for any profit-making activity or commercial gain

- You may freely distribute the URL identifying the publication in the public portal

If you believe that this document breaches copyright please contact us providing details, and we will remove access to the work immediately and investigate your claim 


\title{
Low Cloud Properties Influenced by Cosmic Rays
}

\author{
Nigel D. Marsh and Henrik Svensmark \\ Danish Space Research Institute, Copenhagen, Denmark \\ (Received 18 May 2000; revised manuscript received 15 August 2000)
}

\begin{abstract}
The influence of solar variability on climate is currently uncertain. Recent observations have indicated a possible mechanism via the influence of solar modulated cosmic rays on global cloud cover. Surprisingly the influence of solar variability is strongest in low clouds $(\leq 3 \mathrm{~km})$, which points to a microphysical mechanism involving aerosol formation that is enhanced by ionization due to cosmic rays. If confirmed it suggests that the average state of the heliosphere is important for climate on Earth.
\end{abstract}

PACS numbers: 92.60.Nv, 92.70.Gt, 96.40.Kk

The recent discovery that total cloud cover and solar modulated galactic cosmic ray (GCR) flux are correlated $[1,2]$ suggests that solar variability may be linked to climate variability through a chain involving the solar wind, GCR, and clouds. The solar wind is a continuous flow of energetic charged particles (mainly protons and electrons with energies $\sim \mathrm{KeV}$ ) which are released from the sun as a plasma carrying a fingerprint of the solar magnetic field throughout interplanetary space. Influences from the solar wind are felt at distances well beyond Neptune, possibly up to $200 \mathrm{AU}$ from the sun. This region of space is known as the heliosphere. GCR consists of very energetic particles (mainly protons with typical energies $1-20 \mathrm{GeV}$ ) that originate from stellar processes within our galaxy. Their flux through the solar system is modulated by the shielding effects of the solar wind whose strength is dependent on the level of solar activity. Those incident at the Earth are additionally modulated by the geomagnetic field $[3,4]$ with cutoff rigidities of $15-0.1 \mathrm{GeV}$ from the equator to the geomagnetic poles. The implication from the observed total cloud cover-GCR correlation is that climate on Earth could be influenced by the average state of the heliosphere (heliospheric climate).

Solar forcing of the Earth's climate can be classified into direct and indirect processes. The simplest direct mechanism is through variations in solar radiative output which is known to vary by $0.1 \%$ over the last solar cycle, this corresponds to a change of $0.3 \mathrm{~W} / \mathrm{m}^{2}$ at the top of the Earth's atmosphere. It is currently believed that this effect is too small to have had a dominant influence on surface climate, although variations in solar irradiance may have been larger back in time [5]. Indirect effects include solar induced changes in atmospheric transparency influencing the radiative budget of the planet [1,2,6-9]. One possibility is that changes in the solar output of ultraviolet (UV) radiation affects temperatures in the stratosphere through absorption by ozone, which has the potential to influence the large-scale dynamics of the troposphere $[10,11]$.

The observed GCR-cloud correlation introduces another quite different solar influence with the suggestion that atmospheric ionization produced by GCR $[1,2]$ affects cloud microphysical properties. GCR is the dominant source of atmospheric ionization at altitudes $1-35 \mathrm{~km}$ over the land and $0-35 \mathrm{~km}$ over the oceans with a maximum at $\sim 15 \mathrm{~km}$ due to atmospheric depth. These are regions of the atmosphere in which clouds form. Clouds are of considerable importance for the Earth's radiation budget, although their exact role is currently uncertain. Their influence on the vertically integrated radiative properties of the atmosphere results from cooling through reflection of incoming short wave radiation, and heating through trapping of outgoing long wave radiation. The net radiative impact of a particular cloud is mainly dependent upon its height above the surface and its optical thickness. High optically thin clouds tend to heat while low optically thick clouds tend to cool [12]. The current climatic estimate for the net forcing of the global cloud cover is $\sim 27.7 \mathrm{~W} / \mathrm{m}^{2}$ cooling [12-14]. Thus a significant solar influence on global cloud properties is potentially important for the Earth's radiation budget $[1,2,7]$. However, the spatial properties of cloud formation vary considerably. For example, the physics of high ice clouds is quite different to that for low liquid clouds [15], thus atmospheric ionization need not influence all cloud types. It is imperative to understand which cloud types are influenced by GCR, not only from a radiative point of view but, perhaps more importantly, for identifying a physical mechanism. Since atmospheric ionization from GCR, reaches a maximum at high altitudes and latitudes, intuitively, one might expect this is where clouds would feel the greatest effect. The surprising new result presented here is that only low cloud properties vary with GCR. However, since cloud droplets (in the atmosphere) always condense on an aerosol, this is in agreement with a mechanism where changes in the atmospheric aerosol distribution influences low liquid clouds. It has recently been shown that ionization dominates aerosol production and growth rates when ionization levels are low and trace gas concentrations are high, such as is found in the lower atmosphere [16,17].

State-of-the-art satellite observations of cloud properties are available as monthly averages from the International Satellite Cloud Climate Project (ISCCP) D2 analysis derived from the top of atmosphere (TOA) radiance for the period July 1983 to September 1994 [18-20]. Infrared (IR) measurements (uncertainty 1-2 K [21]) are preferred 
due to their superior spatial and temporal homogeneity over visual observations that can only be detected during daylight. Cloud cover is obtained from an algorithm using the TOA IR statistics to identify the cloudiness on an equal area grid $(280 \mathrm{~km} \times 280 \mathrm{~km})$. Cloud top temperatures (CT) and pressures (CP) are obtained from an ISCCP IR model constrained by water vapor and vertical temperature profiles retrieved from the TIROS observed vertical sounder (TOVS) [20]. CT and CP are found by assuming an opaque blackbody cloud, and adjusting the cloud's pressure level (effectively cloud height) in the model until the reconstructed outgoing IR flux at TOA matches that observed. Based on retrieved $\mathrm{CP}$, clouds are divided into low $>680 \mathrm{hPa}(<3.2 \mathrm{~km})$, middle $=680-440 \mathrm{hPa}$ $(3.2-6.5 \mathrm{~km})$, and high $<440 \mathrm{hPa}(>6.5 \mathrm{~km})$.

Figure 1 indicates that a $2 \%-3 \%$ change in low cloud cover correlates with GCR over the whole period, while the middle and high clouds do not (uncertainties in cloud cover $\leq 1 \%$ [22]). The spatial distribution of this low cloud cover correlation is shown in Fig. 2a. Regions displaying a correlation $r \geq 0.6$ cover a highly significant $15.8 \%$ fraction of the Earth's surface (see Fig. 2 caption). The probability of obtaining such a surface fraction by chance was found to be better than $10^{-3}$ from an ensemble of Monte Carlo simulations. Each member of the ensemble consisted of $N$ independent artificial cloud time series, where $N(\sim 160)$ was the spatial degree of freedom determined from spatial cloud correlations. The most restrictive test was done by generating the artificial cloud series from a Fourier transform of the real cloud data, randomizing the phases, and Fourier transforming back. Note that the high correlation in Fig. 1c, where $r=0.63$ and $r=0.92$ for the 12-month running mean (confidence limits assuming $t$-distribution $<10^{-5}$ ), is obtained by taking the global average of cloud anomalies used in Fig. 2a which reduces fluctuations due to both instrument noise and internal climate variability.

However, at these time scales GCR ionization is not the only mechanism affecting low clouds, there are of course many other decadal processes in the climate system which are important. The small differences in leads and lags are close to the satellites' resolution and one should not expect a perfect correlation. What is surprising is that despite these limitations a signal of solar variability in low cloud cover is dominant at time-scales longer than 1 year. Svensmark [2] argued that there is a better agreement with GCR, rather than solar irradiance, for total cloud cover. This is also true for the low cloud cover in Fig. 1c, which suggests that low cloud cover is responding to cosmic ray ionization in the atmosphere rather than direct changes in solar irradiance.

Currently satellites cannot detect a multilayer cloud, thus high and middle clouds can obscure clouds below. From this point of view low clouds contain the least contaminated signal, giving greater confidence to this result. However, if a cloud is transmissive then the satellite observes both radiation from below the cloud and radiation
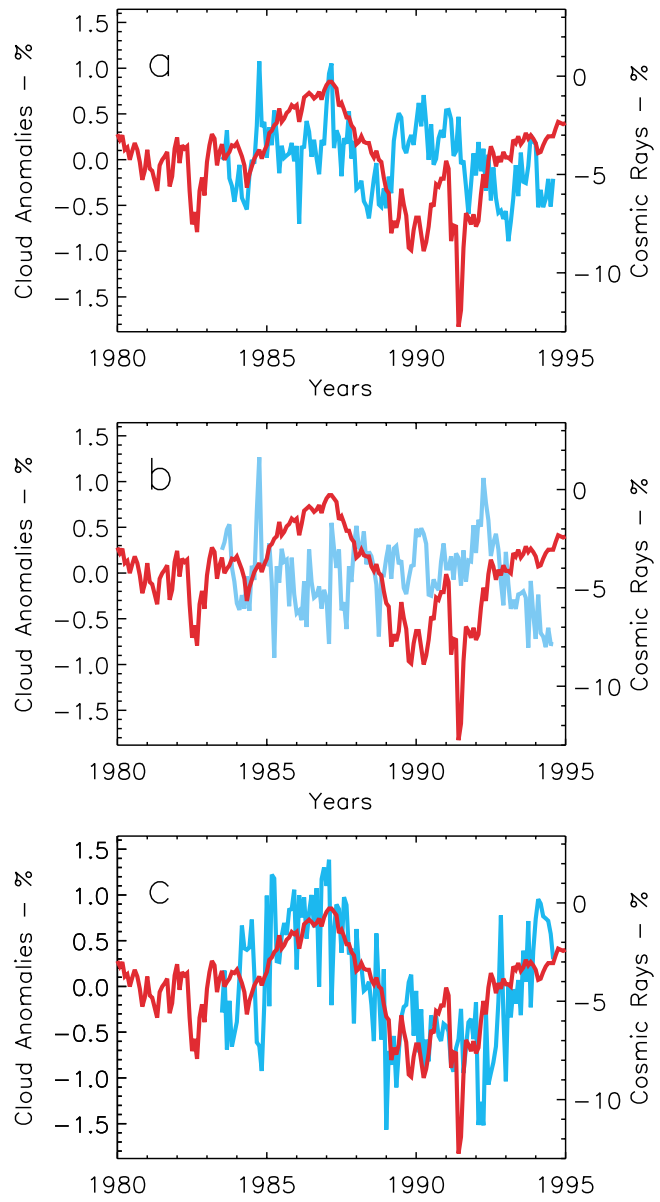

FIG. 1 (color). Global average of monthly cloud anomalies for (a) high $(<440 \mathrm{hPa})$, (b) middle $(440-680 \mathrm{hPa})$, and (c) low $(>680 \mathrm{hPa}$ ) cloud cover (blue). To compute the monthly cloud anomalies the annual cycle is removed by subtracting the climatic monthly average (July 1983-June 1994) from each month on an equal area grid before averaging over the globe. The global average of the annual cycle over this period for high, middle, and low IR detected clouds is $13.5 \%, 19.9 \%$, and $28.7 \%$, respectively. The cosmic rays (red) represent neutron counts observed at Huancayo (cutoff rigidity $12.91 \mathrm{GeV}$ ) and normalized to October 1965.

from the cloud itself. Since ISCCP defines all clouds to be opaque, the CT of transmissive clouds is overestimated, such that their altitude appears lower in the IR model than in reality. For the case of transmissive clouds, CT represents a weighted average based on emissivity of the clouds present in a column scene. However, the long term global trend in low clouds is not explained by an artifact due to mixing with clouds from above since no GCR signal is apparent in the middle and high clouds over the period of observations (Figs. 1a and 1b). But low clouds could be contaminated by overlaying a very thin undetected transmissive cloud, e.g., high thin cirrus, and the signal of solar variability could be due to an undetected high cloud. This is perhaps more intuitive since GCR atmopsheric ionization is greater at higher altitudes, and stratospheric heating 


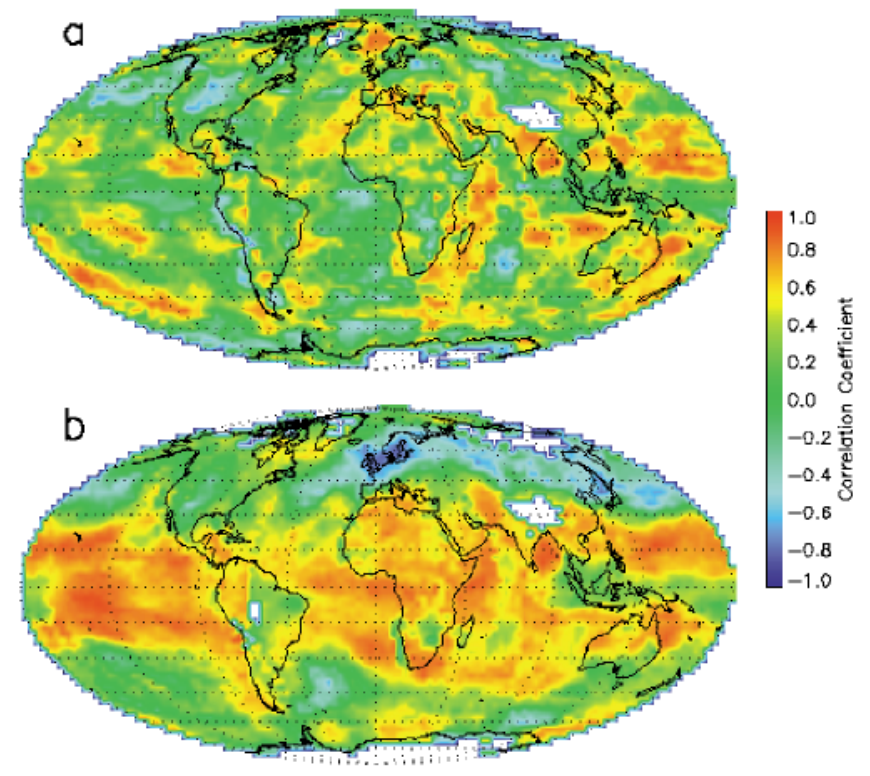

FIG. 2 (color). Global correlation maps of GCR with anomalies of (a) low IR cloud cover and (b) low IR cloud top temperature (CT). The low IR cloud cover is calculated as in Fig. 1c, while the low cloud CT are obtained from the ISCCP IR model. White pixels indicate regions with either no data or an incomplete monthly time series. The correlation coefficients, $r$, are calculated from the 12-month running mean at each grid point. Regions of the Earth with $r \geq 0.6$ are (a) $15.8 \%$ and (b) $34.6 \%$. The probability of obtaining these surface fractions by chance is better than $10^{-3}$.

due to UV possesses a strong solar signal [23]. However, it will be shown that this is not the case.

Although the ISCCP analysis poorly detects a high, very thin cloud, a comparison with high resolution infrared radiation sounder measurements suggests that ISCCP captures the general trends of a high thin cloud [24]. If a solar signal does exist in a high cloud, for whatever reason, one would expect to see a signal in those high clouds that are detected by ISCCP (Fig. 1a). However, no such signal is observed, thus there are good reasons to believe that the long term trends in low cloud cover are due to real low clouds responding to GCR.

The low cloud top temperature parameter also correlates with GCR over large regions of the Earth. Figure $2 b$ reveals a band of significantly high correlation centered around the tropics, while there are no significant correlations for middle and high cloud top temperatures (not shown). The ISCCP IR statistics cannot easily distinguish very low cloud top temperatures, which are relatively warm, from surface temperatures. Thus the modeled surface temperatures (ST) will be contaminated with temperatures from the very low cloud. It is interesting to note that ST contains a very similar GCR correlation map (not shown) as that for low cloud CT in Fig. 2b. The lack of correlation at high latitudes in Fig. $2 b$ is currently not understood, but may be a feature of a possible GCR-cloud mechanism outlined below.
The opaque cloud assumption in the ISCCP IR model excludes microphysical properties and so constrains cloud variability to appear only in cloud "model height," thus introducing an element of artificial variability into CT. Observed properties of low level maritime clouds suggests that they are not opaque [25]. Relaxing the opaque assumption allows for cloud variability to additionally manifest itself through changes in cloud optical density. Cloud optical density depends on processes affecting the cloud droplet size distribution, and cloud vertical extent. Since all atmospheric liquid water droplets form on cloud condensation nuclei $(\mathrm{CCN})$, the droplet size distribution depends on the density of atmospheric aerosols activated as $\mathrm{CCN}$, while cloud thickness is influenced by atmospheric vertical temperature profiles. The abundance of $\mathrm{CCN}$ is determined by both the level of supersaturation and the number of aerosols present in the atmosphere able to act as $\mathrm{CCN}$. Increases in supersaturation, typically between $0.1 \%$ and a few percent, activates increasingly smaller aerosols. A solar signal could enter low cloud properties through influencing atmospheric vertical temperature profiles, water vapor, or aerosol-to-CCN activation processes. In the following it is argued that the latter is a more likely explanation.

Thermodynamic properties of the atmosphere where low clouds form are affected via changes to tropospheric circulation. Studies with general circulation models have indicated that solar induced variability in the stratosphere can influence the vertical circulation of the troposphere $[10,11]$. However, TOVS observations of the vertical profiles of water vapor and temperature demonstrate little correlation with GCR. This suggests that the influence of variability in solar irradiance on local thermodynamic properties in the atmosphere is not responsible for the observed changes in low cloud properties. This might not be surprising given that variability in solar irradiance agrees poorly with changes in low cloud properties [2].

By assuming typical atmospheric water vapor saturation, the abundance of $\mathrm{CCN}$ is determined through properties of the background aerosol size distribution $(\sim 0.01-1.0 \mu \mathrm{m})$. Production of aerosol can be due to many processes involving gas-particle conversion, droplet-particle conversion, i.e., evaporation of water droplets containing dissolved matter, and bulk particles from the surface, e.g., smoke, dust, or pollen [15]. Observations of spectra in regions of low cloud formation indicate that aerosols are produced locally. In the troposphere it has been suggested that ionization contributes to the gas-particle formation of ultrafine $(<0.02 \mu \mathrm{m})$ aerosol. Model studies indicate that this process could contribute a stable concentration of several hundred particles per $\mathrm{cm}^{-3}$ at sizes $>0.02 \mu \mathrm{m}$ [26]. This is comparable to the total number of condensation nuclei in maritime air $\left(\sim 100 \mathrm{~cm}^{-3}\right)$ [15]. Observations of aerosol growth into the aged aerosol distributions generating $\mathrm{CCN}$ have been interpreted to be influenced by the presence of ionization $[16,26,27]$. 
A recent study of ion mediated nucleation by $\mathrm{Yu}$ and Turco [17] indicates that the nucleation rate of ultrafine aerosol is generally limited by ionization from GCRs in the lower maritime atmosphere. In contrast, they show that nucleation in the upper atmosphere is limited by concentrations of trace gases, e.g., $\mathrm{H}_{2} \mathrm{SO}_{4}$. Although it is currently uncertain how the ultrafine aerosol evolves into $\mathrm{CCN}$ it could explain why only low cloud properties are responding to GCR modulation. It is less clear why this modulation should be restricted to lower latitudes, seen particularly in low cloud top temperatures (Fig. 2b), which appears to contradict a larger geomagnetic shielding of cosmic rays at the equator [1]. However, ion mediated nucleation saturates, when levels of ionization are high relative to concentrations of trace gases [17], so a latitudinal dependence of either or both of these could be involved. This is currently an area of further research.

Based on the ISCCP D2 IR cloud data there is a clear correlation between GCR and properties of low clouds in contrast to middle and high clouds. Since the correlation is seen both in low cloud cover and low cloud top temperature, the case for solar induced variability of low clouds is strengthened. Observations of atmospheric parameters from TOVS do not support a solar-cloud mechanism through tropospheric dynamics influenced by UV absorption in the stratosphere. Instead, it is argued that a mechanism involving solar modulated GCR is possible. It has been speculated for some time that ionization is important for aerosol production and growth in the troposphere. Recent studies indicate that ionization is a limiting process for aerosol nucleation in the lower maritime atmosphere, thus it is not unreasonable to imagine that systematic variations in GCR ionization could affect atmospheric aerosols acting as $\mathrm{CCN}$ and hence low cloud properties. If such mechanisms can be confirmed the implications for clouds and climate are far reaching, and suggest that heliospheric climate can influence climate on Earth. Based on observations, Lockwood et al. have shown that, since 1964, the strength of the solar magnetic flux, shielding the Earth from GCR, has increased by $41 \%$ while GCR has decreased by $3.7 \%$ [28]. Further, they claim that the solar magnetic flux has more than doubled over the past century. Based on this doubling, and assuming that a GCR-low cloud mechanism exists, a crude estimate for the century trend in low cloud radiative forcing is a warming of $1.4 \mathrm{Wm}^{-2}$ [29]. Thus, if there is a systematic variation in low cloud properties caused by solar variability it could have important implications for the evolution of Earth's climate.

[1] H. Svensmark and E. Friis-Christensen, J. Atmos Sol.-Terr. Phys. 59, 1225 (1997).
[2] H. Svensmark, Phys. Rev. Lett. 81, 5027 (1998).

[3] D. Lal and B. Peters, Cosmic Ray Produced Radioactivity on the Earth, Encyclopedia of Physics (Springer-Verlag, Berlin, 1967).

[4] J. R. Herman and R. A. Goldberg, Sun, Weather, and Climate, NASA Technical Report SP 426, 1978.

[5] J. Lean, J. Beer, and R. Bradley, Geophys. Res. Lett. 22, 3195 (1992).

[6] E. R. Ney, Nature (London) 183, 451 (1959).

[7] R. Dickinson, Bull. Am. Meteorol. Soc. 56, 1240 (1975).

[8] M. I. Pudovkin and S. Veretenenko, J. Atmos. Terr. Phys. 57, 1349 (1995).

[9] B. A. Tinsley, J. Geomagn. Geoelectr. 48, 165 (1996).

[10] J. D. Haigh, Science 272, 981 (1996).

[11] D. Shindell, D. Rind, N. Balabhandran, J. Lean, and P. Lonergan, Science 284, 305 (1999).

[12] D. L. Hartmann, in Aerosol-Cloud-Climate Interactions, edited by P. V. Hobbs (Academic, San Diego, 1993), p. 151.

[13] V. Ramanathan, R. D. Cess, E.F. Harrison, P. Minnis, B. R. Barkstrom, E. Ahmad, and D. Hartmann, Science 243, 57 (1989).

[14] P. Ardanuy, L. L. Stowe, A. Gruber, and M. Weiss, J. Geophys. Res. 96, 18537 (1991).

[15] H. R. Pruppacher and J. D. Klett, Microphysics of Clouds and Precipitation (Kluwer, Dordrecht, 1997).

[16] F. Yu and R. P. Turco, Geophys. Res. Lett. 27, 883 (2000).

[17] F. Yu and R.P. Turco, in "Contribution to AGU Special Session on Atmospheric Ions: Roles in Aerosol Formation and Chemistry, 2000" (unpublished).

[18] R. A. Schiffer and W. B. Rossow, Bull. Am. Meteorol. Soc. 66, 1498 (1985).

[19] W. B. Rossow and R. A. Schiffer, Bull. Am. Meteorol. Soc. 72, 2 (1991).

[20] W.B. Rossow, A.W. Walker, D.E. Beuschel, and M. D. Roiter, International Satellite Cloud Climatology Project (ISCCP): Documentation of New Cloud Datasets (World Meteorological Organization, Geneva, 1996).

[21] C. Brest, W. B. Rossow, and M. D. Roiter, J. Atmos. Ocean. Technol. 14, 1091 (1997).

[22] W. B. Rossow and B. Cairns, Clim. Change 31, 305 (1995).

[23] H. van Loon and K. Labitzke, J. Atmos. Sol.-Terr. Phys. 61, 53 (1999).

[24] Y. Jin, W. B. Rossow, and D. P. Wylie, J. Clim. 9, 28502 (1999).

[25] A.J. Heymsfield, in Aerosol-Cloud-Climate Interactions (Ref. [12]), p. 97.

[26] R. P. Turco, J. X. Zhao, and F. Yu, Geophys. Res. Lett. 25, 635 (1998).

[27] U. Hõrrak, J. Salm, and H. Tammet, J. Geophys. Res. 103, 13909 (1998).

[28] M. R. Lockwood, R. Stamper, and M. N. Wild, Nature (London) 399, 437 (1999).

[29] N.D. Marsh and H. Svensmark, in Proceedings of ISSI Conference on Solar Variability and Climate, Bern, 1999, edited by R. von Steiger [Space Sci. Rev. (to be published)]. 